Research Article

\title{
Research on Fault Diagnosis Method of Electro-Hydrostatic Actuator
}

\author{
Lei Zhufeng $\mathbb{D}^{1},{ }^{1}$ Qin Lvjun, ${ }^{1}$ Wu Xiaodong, ${ }^{2}$ Jin Wen, ${ }^{1}$ and Wang Caixia ${ }^{1}$ \\ ${ }^{1}$ National Joint Engineering Research Center for Special Pump Technology, Xi'an Aeronautical University, \\ 259 West Second Ring Road, Xi'an, Shaanxi 710077, China \\ ${ }^{2}$ The 49th Research Institute of China Electronics Technology Group Corporation, 969 Longsheng Road, Harbin, \\ Heilongjiang 150028, China \\ Correspondence should be addressed to Lei Zhufeng; leizhufeng@qq.com
}

Received 3 December 2020; Revised 10 January 2021; Accepted 18 January 2021; Published 27 January 2021

Academic Editor: Ke Feng

Copyright (c) 2021 Lei Zhufeng et al. This is an open access article distributed under the Creative Commons Attribution License, which permits unrestricted use, distribution, and reproduction in any medium, provided the original work is properly cited.

\begin{abstract}
Electrohydrostatic actuator is a type of actuator that uses hydraulic energy as the energy transmission carrier, which has the advantages of small size and high power. Since it is commonly used in harsh conditions such as strong vibration, high pressure, and heavy loads, condition monitoring and fault diagnosis of its hydraulic system are particularly important. This paper proposed a novel fault feature extraction method and applied to fault diagnosis of electrohydrostatic actuator. Firstly, the pressure signal of the hydraulic system is decomposed at multiple scales to obtain the center frequency of its maximum energy intrinsic mode component, and the feature data set is constructed based on the statistical features of the time domain. Then, a fault identification model of hydraulic system based on support vector machine is established. Finally, the fault classification and identification results of the hydraulic system are outputted. After a variety of method comparisons, the method proposed in this paper has a fault time ratio accuracy of $96.7 \%$, which provides a basis and a new way for the fault diagnosis of the hydraulic system.
\end{abstract}

\section{Introduction}

The hydraulic transmission has high power density, compact structure, and good dynamic performance. It is widely used in construction machinery, marine vessels, aerospace, and robotics. Long pipeline, poor reliability, large volume, and heavy weight limit the application of traditional centralized electrohydraulic control systems. Distributed electrohydrostatic actuator is small in size, light in weight, and large in output power, which is an important development direction. However, the electrohydrostatic actuator is an important subsystem related to the reliability of the aircraft. How to increase the reliability and service capability of the system while reducing power consumption is a huge challenge it faces.

The hydraulic system is the core component of the electrohydrostatic actuator. The failure of the hydraulic system seriously affects the reliability of the electrohydrostatic actuator and has a great impact on the output power quality. The hydraulic system has problems such as fluid leakage, hydraulic valve failure, and insufficient cooling, which are also important factors affecting system stability. Timely and accurate fault diagnosis and repair of the hydraulic system is a prerequisite for the stable output power of the electrohydrostatic actuator.

Many scholars have conducted a lot of research on the diagnosis of hydraulic system faults. Linaric D et al. [1] applied the neural network model based on the mathematical model of the entire electrohydraulic servo system to perform model-based fault detection and isolation, verified the algorithm by simulation, and achieved significant results. Liu and Jiang [2] improved the application research of fuzzy neural network model in defect detection and classification. Tang et al. [3] through information fusion extracted fault features, through PSO-SVM performed fault classification, and proposed a combined information fusion and PSOSVM multifault diagnosis method for piston pumps, which can effectively identify multiple faults of piston pumps. Gao 
et al. [4] performs the adaptive matching of the noise threshold through median absolute deviation, and a noise suppression method based on Walsh transform is proposed. The method has been verified by numerical simulation and experimental verification of normal pumps and faulty pumps. The method provides a new idea for pump fault diagnosis. Deng et al. [5] in the process of motor bearing fault diagnosis, based on empirical model decomposition and fuzzy information entropy, improved particle swarm optimization algorithm and least squares support vector machine method; an early fault diagnosis method for rotating machinery is proposed and verified by experiments. The multiscale analysis method is an effective signal processing method. The commonly used multiscale processing methods include singular value decomposition (SVD), empirical mode decomposition (EMD), and wavelet transform (WT). In recent years, many scholars have done much research on multiscale analysis method, and they have been applied to the signal processing. Zhang et al. [6] proposed a rotating machinery fault diagnosis method based on Fourier-transform multifilter decomposition. This method uses Fourier-transform multifilter decomposition to decompose the original signal and obtain multiscale frequency domain information. After the feature selection and feature ranking of subsequent subsignals, furthermore, a fault diagnosis method for rotating machinery with higher accuracy and fewer features is obtained. Liang et al. [7] improved the local average decomposition (LMD) by bidirectional long and short-term memory model (Bi-LSTM), which realizes the adaptive expansion of the LMD endpoints, eliminates the LMD endpoint effects, and applies it to the feature extraction of rolling bearings under different loads. In 1998, Huang et al. [8] proposed EMD. EMD is an adaptive decomposition method, and it has been widely used in biomedicine [9-11], speech recognition [12, 13], system modeling [14-16], and process control [17-19]. EMD can decompose the original signal into different frequency bands and enrich the feature information of the original signal by extracting feature information in different frequency bands. EMD has been widely used in production practice. Hu et al. [20] decomposed the children's EEG into various brain wave components by EMD, and 11 different physical quantities are extracted as features in the intrinsic mode function (IMF). Finally, the random forest is used for activity recognition. The experiment verifies the effect of this method on the feasibility of activity identification. Vargas et al. [21] use EMD to decompose the ECG signal into several IMFs and process these IMFs based on the Viterbi algorithm and discrete wavelet transform to realize the noise removal of the ECG signal. Wavelet technology [22] is also an effective multiscale signal processing technology. Therefore, scholars have carried out a lot of research on wavelet technology. Jimenez et al. [23] filter and reduce the noise of wind turbine signals by wavelet transform and perform feature extraction and fault classification. Through the comparison of 6 schemes, the novelty of the method is verified. Based on the wavelet packet transform, a concept of the main frequency band of blasting is defined, which provides an important basis for accurately describing the frequency characteristics of the blasting process [24]. In 2014, Dragomiretskiy et al. [25] proposed the variational mode decomposition method. VMD can separate the components effectively, which is a completely nonrecursive variational mode decomposition model. It finds the center frequency of each IMF by iteratively searching for the optimal solution of the variational model.

Kumar et al. [26] proposed a VMD based on genetic algorithm (GA) and kernel-based mutual information (KEMI) fitness function, which can effectively extract the weak features of multiple defects in bearings. The advantages of VMD in local damage of beam structures are discussed by Mousavi et al. [27], and the peak synchronization between the instantaneous frequency and instantaneous amplitude of the first IMF is considered, which verifies the superiority of VMD in local damage detection.

Although a large number of scholars have conducted indepth research on the fault diagnosis of hydraulic systems, with the continuous development of human society, hydraulic systems continue to be integrated into new use environments, various faults occur frequently, and even multiple faults appear at the same time. With the continuous advancement of computer science and information science, a data-driven hydraulic system fault diagnosis method using hydraulic system monitoring data has emerged. Finding fault characteristics from hydraulic system monitoring signals and accurately identifying fault types is the key study of this article.

This paper proposed a feature extraction method based on multiscale signal processing and applied it to the fault diagnosis of the hydraulic system to improve the fault identification accuracy and efficiency. Firstly, a new type of variational modal decomposition method is given. Secondly, we introduced the hydraulic system test platform on which the data collection of this article is based, introduced the composition and hydraulic principle of the hydraulic system test platform, and conducted data acquisition of hydraulic system pressure signal under four failure modes, and performed multiscale decomposition of the original signal; the maximum energy intrinsic mode component central frequency of pressure signal is obtained, the feature data set is constructed with the statistical features based on the time domain, and the pattern recognition is performed. Finally, the influence of multiple feature data set construction methods on the fault recognition accuracy of hydraulic system is discussed.

\section{Establishment of Fault Diagnosis Model for Hydraulic Power Generation System}

2.1. Variational Modal Decomposition. VMD is a completely nonrecursive variational modal decomposition model. In the process of iteratively searching for the optimal solution of the variational model, the center frequency and bandwidth of each decomposition and separation are determined, so each component of the signal can be effectively separated and the adaptive frequency domain division of the signal can be realized. Since the signal is decomposited into nonrecursive and variational modal components by VMD, it 
shows better noise and sampling rate robustness than the recursive screening modal method of EMD.

VMD is based on the mixing-frequency variational problem-solving process, Hilbert transform, and classical Wiener filtering. In the process of iterative search for the optimal solution of the variational model, the center frequency and bandwidth of each decomposition and separation can be determined, which can effectively separate the components of the signal and realize the adaptive frequency domain division of the signal, so the signal can be decomposed into the sparsity component, which is a new signal decomposition estimation method. The variational modal decomposition algorithm redefines an AM-FM as an intrinsic modal function, and its expression is

$$
u_{k}(t)=A_{k}(t) \cos \left(\phi_{k}(t)\right)
$$

where $A_{k}(t)$ is amplitude of $u_{k}(t), A_{k}(t) \geq 0 . \omega_{k}(t)=\varphi_{k}^{\prime}(t)$, $\omega_{k}(t)$ is the frequency of $u_{k}(t)$. The phase $\varphi_{k}(t)$ is a nondecreasing function. In the interval $[t-\delta, t+\delta], u_{k}(t)$ is a harmonic signal with amplitude $A_{k}(t)$, where $\delta=2 \pi / \phi_{k}^{\prime}(t)$.

VMD is different from the iterative screening process used in the empirical mode decomposition method when obtaining the IMF. It is a nonrecursive method based on the principle of the variational model. The signal is decomposed into sparse components; at the same time, the center frequency and bandwidth of each IMF are determined in the process of iteratively solving the optimal solution of the constrained variational model.

The variational constraint model is shown as follows:

$$
\begin{aligned}
& \min _{\left\{u_{k}\right\},\left\{\omega_{k}\right\}}\left\{\sum_{k}\left\|\partial_{t}\left[\left(\delta(t)+\frac{j}{\pi t}\right) \times u_{k}(t)\right] e^{-j \omega k t}\right\|_{2}^{2}\right\}, \\
& \text { s.t. } \sum_{k} u_{k}=f,
\end{aligned}
$$

where $\left\{u_{k}\right\}:=\left\{u_{1}, u_{2}, \ldots, u_{K}\right\}$ represents K IMF components, $\left\{\omega_{k}\right\}:=\left\{\omega_{1}, \omega_{2}, \ldots, \omega_{K}\right\}$ represents the center frequency of each IMF, and $\sum_{k}$ : $=\sum_{k=1}^{K}$ represents the sum of all modes.

After the original signal is decomposed by VMD, the center frequency of the maximum energy of the IMF can be obtained according to the following formula:

$$
\mathrm{CIMF}=\max _{1 \leq i \leq n-1}\left[E\left(\mathrm{IMF}_{i}, \mathrm{IMF}_{i+1}\right)\right],
$$

where CIMF is the maximum energy of the IMF, $\mathrm{IMF}_{i}$ is the $i^{\text {th }} \mathrm{IMF}$, and $\mathrm{E}\left(\mathrm{IMF}_{i}\right)$ is energy of the $i^{\text {th }} \mathrm{IMF}$.

The maximum energy of the IMF is the component with the largest energy in the process of multiscale analysis of the original signal. It occupies a large proportion of the information implicit in the original signal and is the main component in the original signal. It can be seen in Figure 1 [28]. IMF.

As shown in Figure 1, IMF8 is maximum energy of the

2.2. Support Vector Machine. SVM is an effective tool for solving classification and regression problems. The linear classifier with the largest interval defined in the feature space is the basic model of SVM [29]. Nonlinear SVM is an improvement and general case of linear separable SVM. Linearly separable SVM is a linear classifier that uses hard interval maximization to learn when the training data are linearly separable. Linear SVM is a linear classifier that uses soft interval to maximize learning when the training data are approximately linearly separable. Nonlinear SVM is a nonlinear classifier that uses nuclear techniques to learn when the training data are linearly inseparable. Therefore, the SVM introduced with the kernel technique has become a substantial nonlinear classifier.

Suppose the training set of a feature space is $D=\left\{\left(x_{1}, y_{1}\right),\left(x_{2}, y_{2}\right), \ldots,\left(x_{m}, y_{m}\right)\right\} ; \quad$ among them, $x_{i} \in \chi=\mathfrak{R}^{n}, y_{i} \in y=\{+1,-1\}, i=1,2, \ldots, N, x_{i}$ is a feature vector and $y_{i}$ is a label of $x_{i}$.

The classification hyperplane is

$$
h(x)=\omega \cdot x+b,
$$

where $x$ is the input vector, $\omega$ is the normal vector, and $b$ is the intercept.

The classification decision function is

$$
\begin{aligned}
& \text { Sign }(h((x)), \\
& \left\{\begin{array}{l}
h(x)>0, y_{i}=1, \\
h(x)<0, y_{i}=-1 .
\end{array}\right.
\end{aligned}
$$

SVM is to find an optimal hyperplane in the feature space so that the data set is divided into different classes, and the interval between the closest points is maximized. There are infinitely many solutions for its classification hyperplane. Using interval maximization to solve the classification hyperplane, the solution at this time is unique.

A cost factor $\xi_{i}$ is paid for each slack variable $\xi_{i}$, and then formula 15 is transformed into

$$
\frac{1}{2}\|\omega\|^{2}+C \sum_{i=1}^{N} \xi_{i}
$$

where $C>0$ is the penalty parameter.

The function of $\mathrm{C}$ is to reconcile the coefficients of formula 6 . When $\mathrm{C}$ increases, the penalty for misclassification increases, and on the contrary, the penalty for misclassification decreases.

Formula 6 is to make $(1 / 2)\|\omega\|^{2}$ as small as possible, that is, the interval is maximized while the points of misclassification are minimized.

Construct the Lagrangian function as follows:

$$
L(\omega, b, \alpha)=\frac{1}{2}\|\omega\|^{2}-\sum_{i=1}^{N} \alpha_{i}\left[y_{i}\left(\omega \cdot x_{i}+b\right)-1+\xi_{i}\right]+C \sum_{i=1}^{N} \alpha_{i},
$$

where $\alpha_{i} \geq 0$ is Lagrange multiplier, $i=1,2, \ldots, N, N$ is the total number of samples in the sample set.

According to the KarushKuhnTucker condition, the partial differentiation of $\omega, b$, and $\xi$ is obtained and we set 

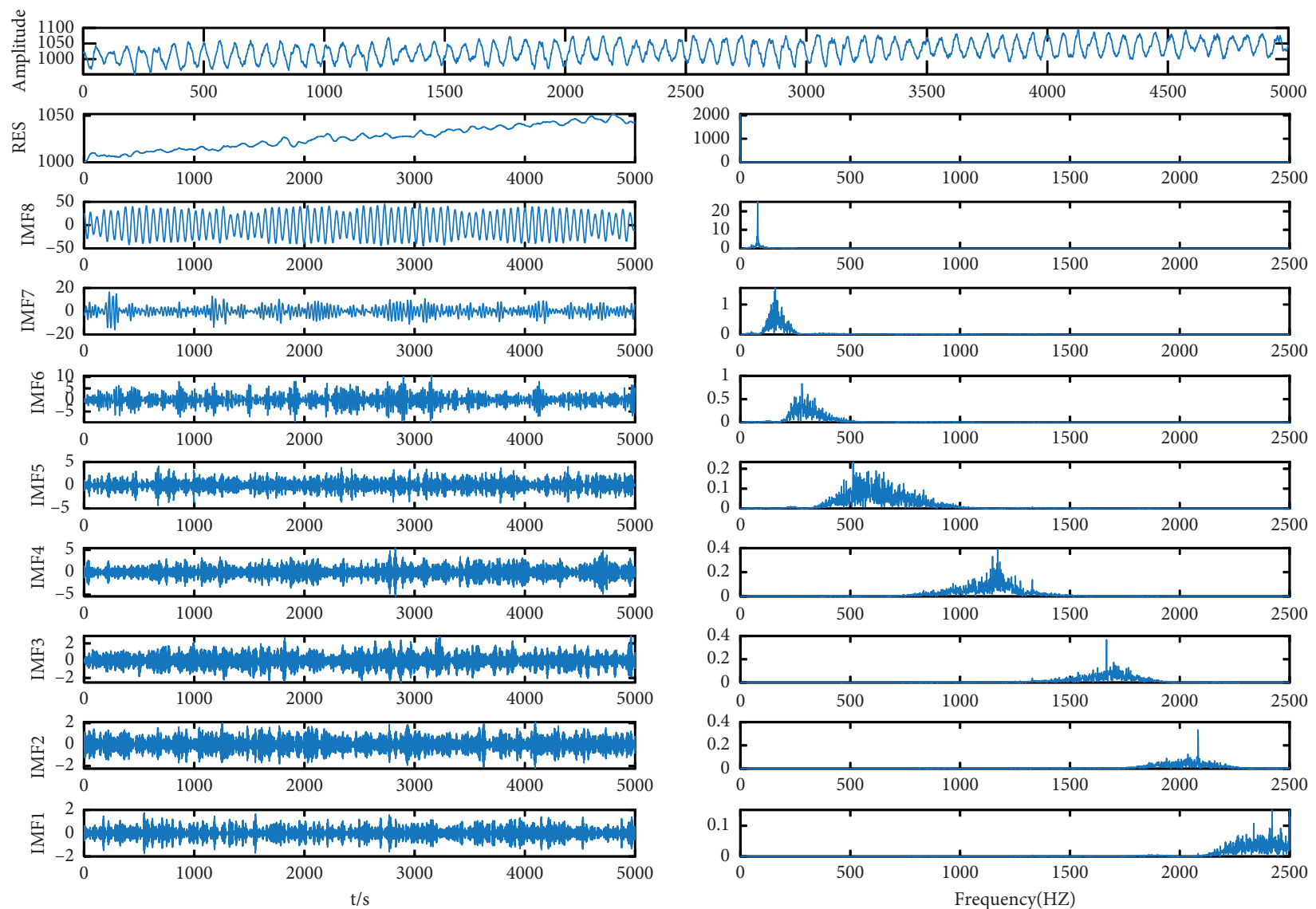

Figure 1: VMD decomposition example.

them equal to 0 , and thus the following formula can be obtained:

$$
\begin{gathered}
\omega=\sum_{i=1}^{N} y_{i} \alpha_{i} x_{i}, \\
-\sum_{i=1}^{N} y_{i} \alpha_{i}=0,
\end{gathered}
$$

where $\quad \alpha_{i}\left(y_{i}\left(\omega \cdot x_{i}+b\right)-1\right)=0, \quad i=1,2, \ldots, N$, $y_{i}\left(\omega \cdot x_{i}+b\right)-1 \geq 0, i=1,2, \ldots, N, \alpha_{i} \geq 0, i=1,2, \ldots, N$.

Thus, the separating hyperplane can be written as

$$
\sum_{i=1}^{N} \alpha_{i} y_{i}\left(x \cdot x_{i}\right)+b=0
$$

The classification decision function can be written as

$$
f(x)=\operatorname{sign}\left[\sum_{i=1}^{N} \alpha_{i} y_{i}\left(x \cdot x_{i}\right)+b\right],
$$

where $b=y_{j}-\sum_{i=1}^{N} \alpha_{i} y_{i}\left(x \cdot x_{i}\right), y_{j}$ is support vector.

For linear classification problems, linear support vector machines are an effective method. However, in actual data, most of the data are linear and inseparable. In order to transform nonlinear problems into linear problems and linear inseparable problems into linear separable problems, linear support vector machines are suitable for the linear inseparable data problems that are often encountered in practical problems.

Let input space $\chi$ be a subset or discrete set of Euclidean space $\Re_{n}$ and feature space (Hilbert space) $\mathscr{H}$, if there is a mapping from $\chi$ to $\mathscr{H}$ :

$$
\varphi(x): \chi \longrightarrow \mathscr{H} .
$$

Such that for all $x_{i}, x_{j} \in \chi$, the function $k\left(x_{i}, x_{j}\right)$ satisfies the following condition:

$$
K\left(x_{i}, x_{j}\right)=\varphi\left(x_{i}\right) \cdot \varphi\left(x_{j}\right) .
$$

Then, $k\left(x_{i}, x_{j}\right)$ is the kernel function.where $\varphi(x)$ is the mapping function and $\varphi\left(x_{i}\right) \cdot \varphi\left(x_{j}\right)$ is the inner product of $\varphi$ $\left(x_{i}\right)$ and $\varphi\left(x_{j}\right)$.

At this point, the objective function becomes

$$
W(\alpha)=\frac{1}{2} \sum_{i=1}^{N} \sum_{j=1}^{N} \alpha_{i} \alpha_{j} y_{i} y_{j} K\left(x_{i}, x_{j}\right)-\sum_{i=1}^{N} \alpha_{i},
$$

where $\alpha_{i}>0$ is Lagrangian multiplier and $N$ is total number of samples in the sample set.

The inner product in the classification decision function can be replaced by the kernel function, and then the classification decision function becomes 


$$
\begin{aligned}
f(x) & =\operatorname{sign}\left[\sum_{i=1}^{N} a_{i} y_{i} K\left(x_{i}, x\right)+b\right], \\
K\left(x_{i}, x\right) & =\exp \left(\frac{-\left\|x_{i}-x\right\|^{2}}{2 g^{2}}\right) .
\end{aligned}
$$

The following formula is a Gaussian radial basis function classifier, and its classification decision function is

$$
f(x)=\operatorname{sign}\left[\sum_{i=1}^{N} a_{i} y_{i} \exp \left(\frac{-\left\|x_{i}-x\right\|^{2}}{2 g^{2}}\right)+b\right],
$$

where $g$ is the kernel function parameter.

2.3. Integrated Algorithm Flow and Steps. As is shown in Figure 2, the pressure signal collected in the hydraulic system is first used for signal noise reduction, and the noisereduced signal is extracted by two methods. One is the feature extraction based on time series signal statistical features, including peak-to-peak value, standard deviation, variance, mean, peak value, skewness, and kurtosis; the second is to perform VMD on the noise-reduced signal to obtain the center frequency of maximum energy intrinsic mode component. Taking the center frequency of the maximum energy intrinsic mode component as the eigenvalue, combine the statistical features to combine the feature data set. Then, the combined feature data set is divided into a training set and a test set. Through the training set, a fault recognition model which is based on the support vector machine-based hydraulic system is constructed, and the model parameters are optimized. The test set is used to verify the accuracy and efficiency of the optimized fault recognition model. Finally, the classification and recognition results are output.

\section{Experimental Analysis}

3.1. Hydraulic Test Rig. In order to verify the reliability and robustness of the method described in this article, we developed a hydraulic test rig, as shown in Figure 3; it consists of hydraulic pump station, filter temperature control system, test console, hydraulic component test platform, test data acquisition system, and electrical control system, etc. The test rig uses the pump as the drive and the motor as the load. The performance of the hydraulic components can be tested by replacing different components on the hydraulic test rig. The performance parameters of the test rig are shown in Table 1.

The principle of the hydraulic test rig is shown in Figure 4.

As shown in Figure 4, 1 is the hydraulic pump group, 2 is the cooling system, 3 is the flow meter, 4 is the one-way valve, 5 is the pressure sensor, 6 is the torque speed tester, 7 is the loading pump, 8 is the motor, 9 is the temperature sensor, 10 is the overflow valve, 11 is the reversing valve, and 12 is the accumulator. The pump 1 is driven by an electric motor, and the system pressure is adjusted through a one- way valve group. The state of different hydraulic components can be monitored through the pressure and temperature values of different positions.

3.2. Failure Category of Hydraulic Power Generation System. In the hydraulic system, different hydraulic components will have different types of failures under different working conditions, even the same hydraulic components will have different types of failures under different working conditions, and the solutions are also different for different types of failures.

The fluid characteristics in the hydraulic system are different from the mechanical vibration characteristics, so it is impossible to perform fault diagnosis of the hydraulic system by monitoring the vibration of the system components.

This paper simulates the fluid leakage, hydraulic valve failure, accumulator failure, and other failure forms of the hydraulic system in the unstable state and the stable state of the system as shown in Table 2. Among them, 1 indicates the best state and 0 indicates the failure state.

As shown in Figure 5, using a hydraulic test rig, the inlet pressure of the check valve 3 was collected while simulating these failures. The sampling frequency was $100 \mathrm{~Hz}$ and the sampling time was 8 seconds. The fault diagnosis model of hydraulic system is verified through four fault types.

\subsection{Fault Identification Based on the Maximum Energy Center} Frequency. Firstly, the collected pressure signal noise reduction is made, and the maximum energy intrinsic mode component of each signal is calculated. The center frequency of the maximum energy intrinsic mode component is selected as the feature, and the statistical feature of the original signal based on support vector machine is combined with for classification calculation. Statistical features include peak-topeak value, standard deviation, variance, mean, peak value, skewness, and kurtosis. $60 \%$ of the feature set is defined as the training set, and $40 \%$ is defined as the test set. According to the method of document 28 , the original signal in this paper is decomposed by VMD to obtain 9 IMFs. The parameter C in SVM is 13.368 and $g$ is 0.01 . The classification result is shown in Figure 6.

It can be seen from Figure 6 that the prediction accuracy of the hydraulic power generation system fault diagnosis model based on the maximum energy center frequency can reach $96.7 \%$.

\section{Discussion}

In order to verify the feasibility and effectiveness of using the multiscale features of variational modal decomposition to construct the feature data set proposed in this paper, a variety of methods are used for comparative analysis, including only using statistical features for fault identification, only using the maximum energy intrinsic mode component center frequency for fault identification and classification model, and using Gaussian kernel function and Sigmoid kernel function for fault recognition. The recognition results of multiple methods are 


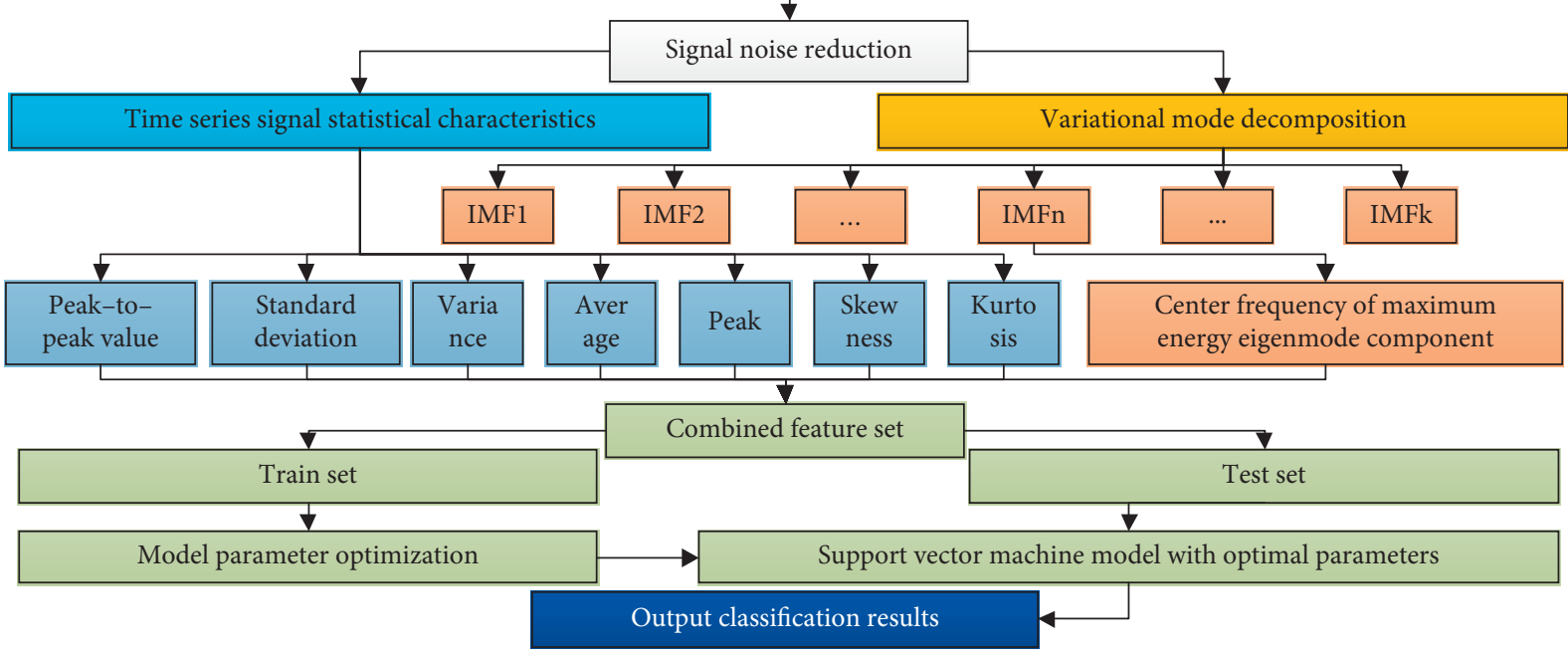

FIGURE 2: Integrated algorithm flow.

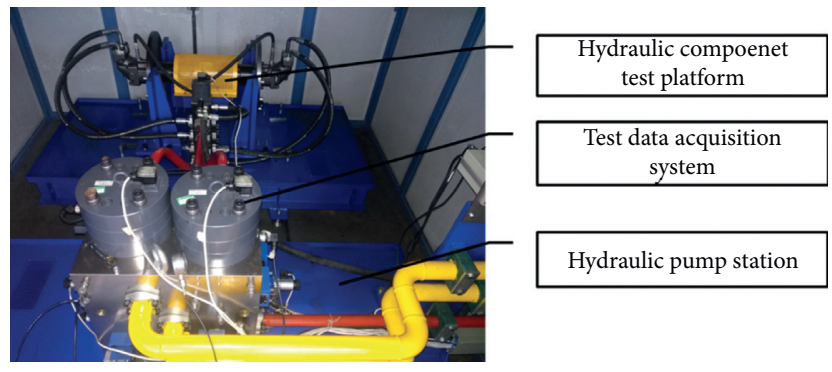

Figure 3: Hydraulic test rig.

TABLE 1: The performance parameters of the test rig.

\begin{tabular}{lccccc}
\hline \multirow{2}{*}{$\begin{array}{l}\text { PerformanceO } \\
\text { Operating temperature }\left({ }^{\circ} \mathrm{C}\right)\end{array}$} & Flow meter & Pressure sensor & Torque meter $(\mathrm{N} \bullet \mathrm{m})$ \\
\hline $\begin{array}{l}\text { Target } \\
\text { Performance }\end{array}$ & Operating pressure (bar) & Rated flow $(\mathrm{L} / \mathrm{min})$ & Total power $(\mathrm{KW})$ & $\pm 5 \% \mathrm{FS}$ & Noise $(\mathrm{dB})$ \\
Target & $0-420$ & 320 & 215 & $\leq 85 \mathrm{~dB}$ & $\leq 2000$ \\
\hline
\end{tabular}

compared so that the best model for fault diagnosis of hydraulic power generation system is selected.

The comparison result is shown in Table 3, as shown in Figure 7.

It can be seen from Table 3 and Figure 7 that the accuracy of fault identification only using the statistical feature of the fault information feature data set is only $56.7 \%$, and the accuracy of only using the central frequency of the maximum energy intrinsic mode component for fault identification reaches $93.3 \%$. The accuracy of fault identification by integrating the feature data sets of the two feature combinations has been greatly improved, reaching $96.7 \%$.

Since the Gaussian kernel function maps the feature data set to high dimensions, the indivisible data become separable and the Sigmoid kernel function makes the support vector machine model a multilayer perceptron neural network. Therefore, the classification performance of the Gaussian kernel function used in the classification model is obviously worse than the performance of the Sigmoid kernel function.

The center frequency of the maximum energy intrinsic mode component is a kind of multiscale spectral information, which is essentially different from the statistical information extracted based on time-domain signals. Using the center frequency of the maximum energy intrinsic mode component as a feature to construct a feature data set has a positive impact on the accuracy of electrohydrostatic actuator fault recognition. 


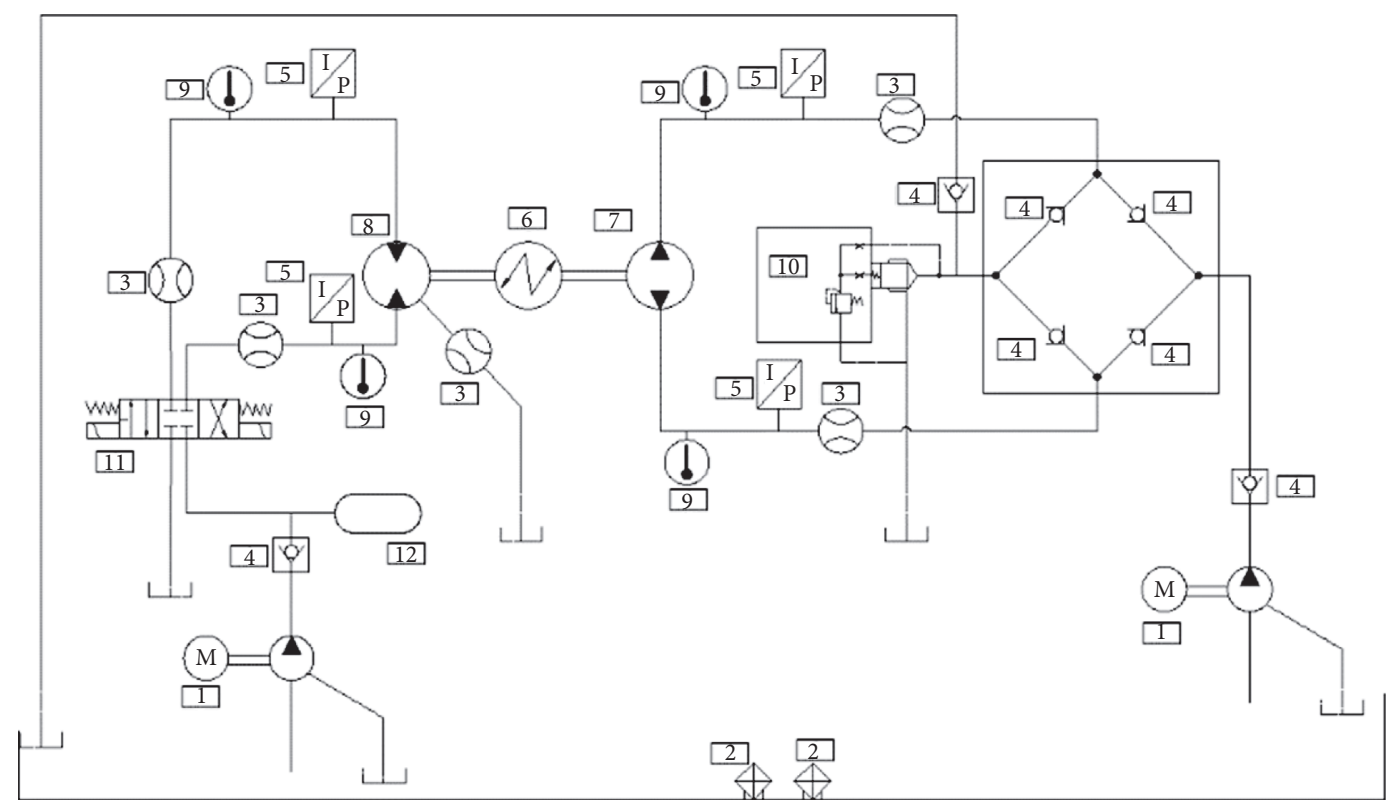

FIgURE 4: The principle of the hydraulic test rig.

TABLE 2: Simulated fault information.

\begin{tabular}{lcccc}
\hline Fault type & Valve status & Pump leak & Accumulator failure & System status \\
\hline Valve status & 0 & 1 & 1 & 1 \\
Pump leak & 1 & 0 & 1 & 1 \\
Accumulator failure & 1 & 1 & 0 & 1 \\
System status & 1 & 1 & 1 & 0 \\
\hline
\end{tabular}

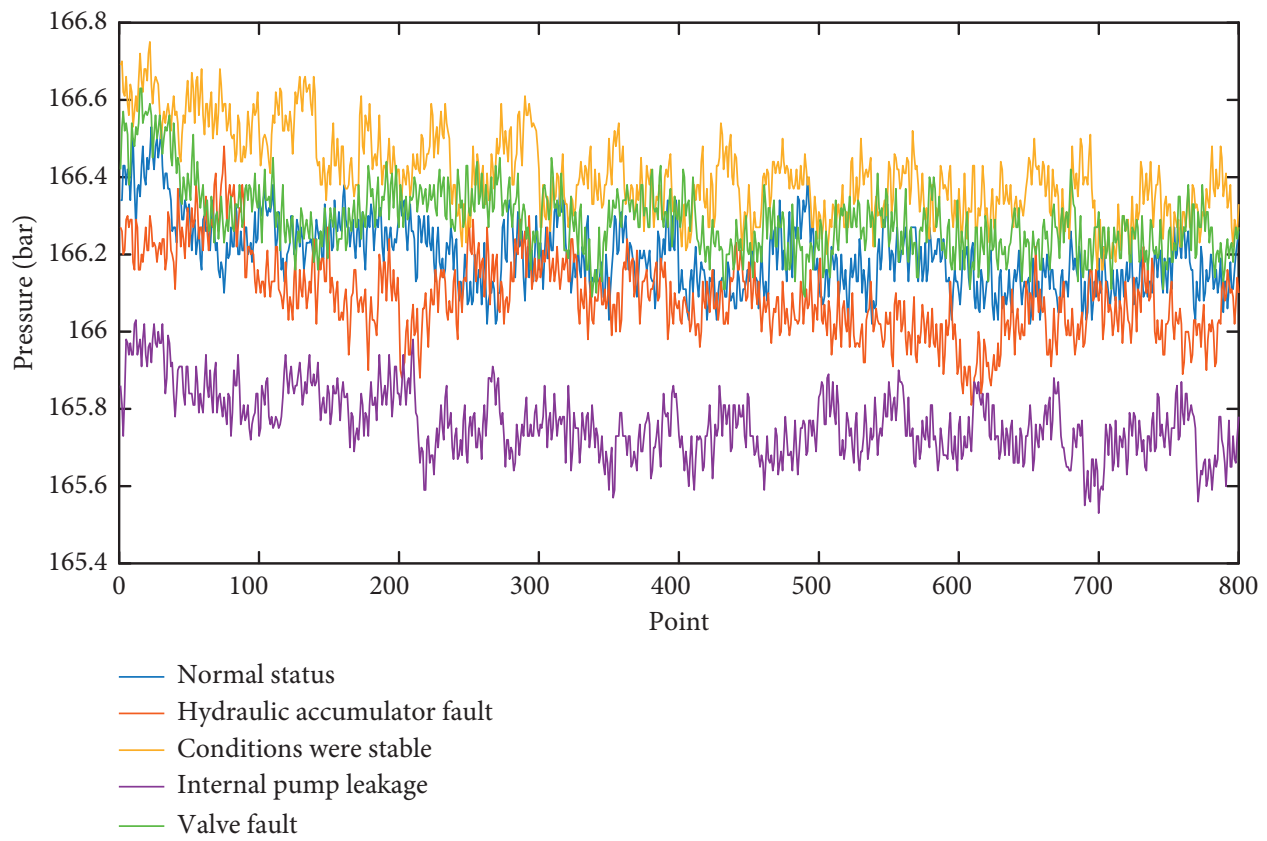

FIGURE 5: The pressure signal curve under five different fault conditions collected by the hydraulic system. 


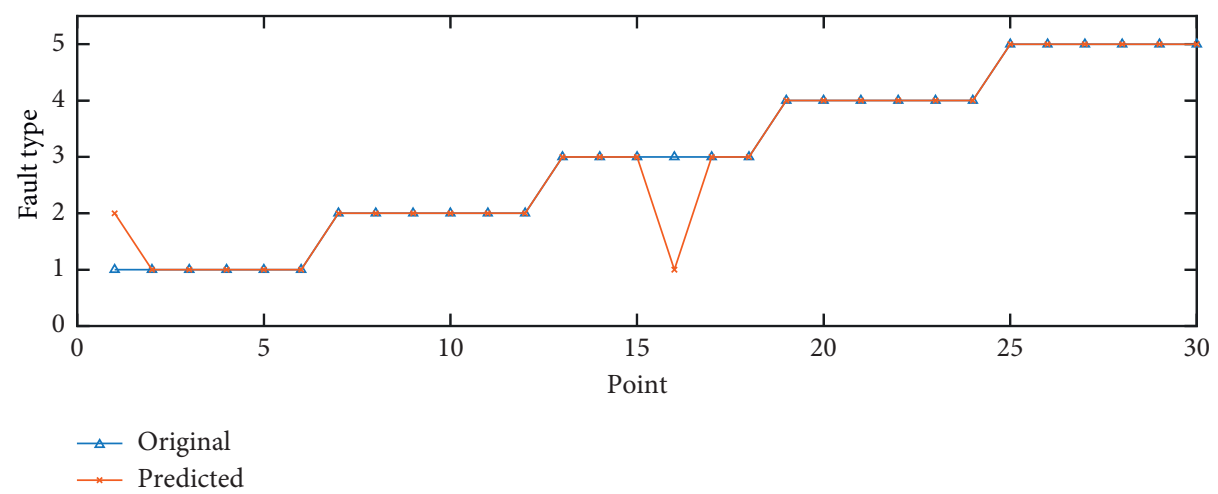

Figure 6: Result of fault identification.

TABLE 3: Various methods of fault identification results.

\begin{tabular}{lcccccc}
\hline \multirow{2}{*}{ Method } & \multicolumn{2}{c}{$\begin{array}{c}\text { Comprehensive feature } \\
\text { data set }\end{array}$} & \multicolumn{2}{c}{$\begin{array}{c}\text { Center frequency feature } \\
\text { data set }\end{array}$} & \multicolumn{2}{c}{ Statistical feature data set } \\
\hline $\begin{array}{l}\text { Kernel function type } \\
\text { Results }\end{array}$ & Sigmoid & Gauss & Sigmoid & Gauss & Sigmoid (\%) & Gauss (\%) \\
\hline
\end{tabular}

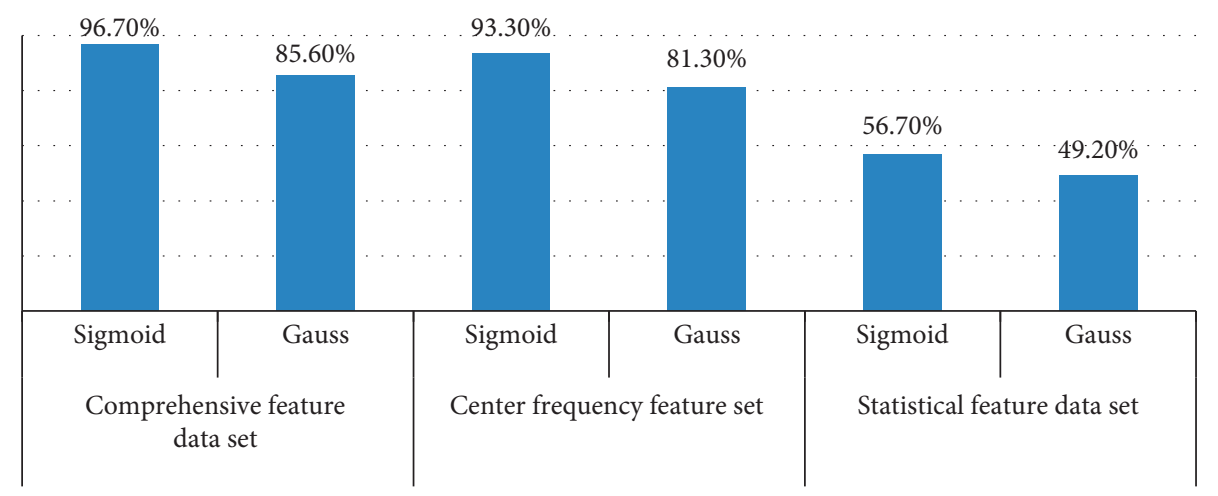

FIGURE 7: Fault identification results of various methods.

\section{Conclusions}

This paper proposes a multi-scale feature extraction method based on variational modal decomposition. The central frequency of the maximum energy intrinsic mode component and the statistical features based on the time-domain signal are combined to construct a feature data set and establish the fault diagnosis model of the hydraulic system, which greatly improves the accuracy of the model for fault identification of the electro-hydrostatic actuator. It can be seen that the feature data set constructed with the multiscale features obtained by the variational modal decomposition method described in this article can more clearly reflect the characteristics of the fault information, which provides a basis for the fault diagnosis of the hydraulic system and provides a new way for electro-hydrostatic actuator fault diagnosis.

\section{Data Availability}

The data used to support the findings of this article are provided within the article.

\section{Conflicts of Interest}

The authors declare that they have no conflicts of interest.

\section{Authors' Contributions}

Jin Wen was involved in the project administration; Lei Zhufeng wrote and prepared the original draft; Wu Xiaodong investigated the study; Qin Lvjun and Wang Caixia wrote, reviewed, and edited the manuscript.

\section{Acknowledgments}

Wei Hongbo and Zhang Yifei are acknowledged for their valuable technical support. This work was financially supported by the Science Foundation of Xi'an Aeronautical University (no. 2020KY0223) and the National Natural Science Foundation of China (no. 11902236).

\section{References}

[1] D. Linaric and V. Koroman, "Fault diagnosis of a hydraulic actuator using neural network," in Proceedings of the IEEE 
International Conference on Industrial Technology, pp. 108111, Maribor, SI, Slovenia, December 2003.

[2] C. Liu and H. Jiang, "A seventh-order model for dynamic response of an electro-hydraulic servo valve," Chinese Journal of Aeronautics, vol. 27, no. 6, pp. 1605-1611, 2014.

[3] H. Tang, Z. Wang, and Y. Wu, "A multi-fault diagnosis method for piston pump in construction machinery based on information fusion and PSO-SVM," Journal of Vibro Engineering, vol. 21, pp. 1904-1916, 2019.

[4] Q. Gao, H.-S. Tang, J.-W. Xiang, and Y. Zhong, "A multisensor fault detection strategy for axial piston pump using the Walsh transform method," International Journal of Distributed Sensor Networks, vol. 14, no. 4, Article ID $155014771877253,2018$.

[5] W. Deng, R. Yao, H. Zhao, X. Yang, and G. Li, "A novel intelligent diagnosis method using optimal LS-SVM with improved PSO algorithm," Soft Computing, vol. 23, no. 7, pp. 2445-2462, 2019.

[6] C. Zhang, L. Kong, Q. Xu, K. Zhou, and H. Pan, "Fault diagnosis of key components in the rotating machinery based on Fourier transform multi-filter decomposition and optimized LightGBM," Measurement Science and Technology, vol. 32, no. 1, Article ID 015004, 2021.

[7] J. Liang, L. Wang, J. Wu, Z. Liu, and G. Yu, "Elimination of end effects in LMD by Bi-LSTM regression network and applications for rolling element bearings characteristic extraction under different loading conditions," Digital Signal Processing, vol. 107, Article ID 102881, 2020.

[8] N. E. Huang, Z. Shen, S. R. Long et al., "The empirical mode decomposition and the Hilbert spectrum for nonlinear and non-stationary time series analysis," Proceedings of the Royal Society of London. Series A: Mathematical, Physical and Engineering Sciences, vol. 454, no. 1971, pp. 903-995, 1998.

[9] M. Li, X. Sun, and W. Chen, "Patient-specific seizure detection method using nonlinear mode decomposition for long-term EEG signals," Medical \& Biological Engineering \& Computing, vol. 58, no. 12, pp. 3075-3088, 2020.

[10] S. Yang, B. Li, Y. Zhang et al., "Selection of features for patient-independent detection of seizure events using scalp EEG signals," Computers in Biology and Medicine, vol. 119, Article ID 103671, 2020.

[11] S. Xie and S. Krishnan, "Feature extraction of epileptic EEG using wavelet power spectra and functional PCA," in Proceedings of the 41st Annual International Conference of the IEEE Engineering in Medicine and Biology Society, pp. 25512554, Berlin, Germany, July 2019.

[12] R. Hemavathi and R. Kumaraswamy, "A study on unsupervised monaural reverberant speech separation," International Journal of Speech Technology, vol. 23, no. 2, pp. 451-457, 2020.

[13] R. Tejus, Y. Nishant, S. Moin et al., "Role of source separation using combined RPCA and block thresholding for effective speaker identification in multi source environment," in Proceedings of the 2017 2nd IEEE International Conference on Recent Trends in Electronics, Information \& Communication Technology (RTEICT), pp. 1253-1256, Bangalore, India, January 2017.

[14] Y. Xu and G. Yang, "A short-term wind speed forecasting hybrid model based on empirical mode decomposition and multiple kernel learning," Complexity, vol. 2020, Article ID 8811407, 13 pages, 2020.

[15] S. Li, X. Liu, and A. Lin, "Fractional frequency hybrid model based on EEMD for financial time series forecasting," Communications in Nonlinear Science and Numerical Simulation, vol. 89, Article ID 105281, 2020.
[16] W. Ding and F. Meng, "Point and interval forecasting for wind speed based on linear component extraction," Applied Soft Computing, vol. 93, Article ID 106350, 2020.

[17] K. H. Choi, "Low-frequency pressure fluctuations in an external-loop airlift reactor," Korean Chemical Engineering Research, vol. 58, pp. 665-674, 2020.

[18] Z. Lei and W. Su, "Mold level predict of continuous casting using hybrid EMD-SVR-GA algorithm," Processes, vol. 7, no. 3, p. 177, 2019.

[19] Y. Zhang, X. Yang, F. Zhu et al., "Characteristics of non-linear dynamics and energy transfer in a vibration gas-solid fluidized bed by using Hilbert-Huang transform," Powder Technology, vol. 344, pp. 970-980, 2019.

[20] L. Hu, K. Zhao, X. Zhou, B. W.-K. Ling, and G. Liao, "Empirical mode decomposition based multi-modal activity recognition," Sensors, vol. 20, no. 21, p. 6055, 2020.

[21] R. N. Vargas and A. C. P. Veiga, "Empirical mode decomposition, viterbi and wavelets applied to electrocardiogram noise removal," Circuits System Signal Process, vol. 28, 2020.

[22] X. Chiementin, B. Kilundu, L. Rasolofondraibe, S. Crequy, and B. Pottier, "Performance of wavelet denoising in vibration analysis: highlighting," Journal of Vibration and Control, vol. 18, no. 6, pp. 850-858, 2012.

[23] A. A. Jimenez, L. Zhang, C. Q. G. Munoz et al., "Maintenance management based on Machine Learning and nonlinear features in wind turbines," Renewable Energy, vol. 146, pp. 316-328, 2020.

[24] G. Chen, Q.-Y. Li, D.-Q. Li, Z.-Y. Wu, and Y. Liu, "Main frequency band of blast vibration signal based on wavelet packet transform," Applied Mathematical Modelling, vol. 74, pp. 569-585, 2019.

[25] K. Dragomiretskiy and D. Zosso, "Variational mode decomposition," IEEE Transactions on Signal Processing, vol. 62, no. 3, pp. 531-544, 2014.

[26] A. Kumar, Y. Zhou, and J. Xiang, "Optimization of VMD using kernel-based mutual information for the extraction of weak features to detect bearing defects," Measurement, vol. 168, Article ID 108402, 2021.

[27] M. Mousavi, D. Holloway, J. C. Olivier, and A. H. Gandomi, "Beam damage detection using synchronisation of peaks in instantaneous frequency and amplitude of vibration data," Measurement, vol. 168, Article ID 108297, 2021.

[28] W. Su and Z. Lei, "Mold-level prediction based on long shortterm memory model and multi-mode decomposition with mutual information entropy," Advances in Mechanical Engineering, vol. 11, no. 12, Article ID 168781401989443, 2019.

[29] X. Zhang and J. Zhou, "Multi-fault diagnosis for rolling element bearings based on ensemble empirical mode decomposition and optimized support vector machines," Mechanical Systems and Signal Processing, vol. 41, no. 1-2, pp. 127-140, 2013. 